\title{
Implementing an Academic Task Manager: Findings from a Pilot Study
}

\author{
Senanu Okuboyejo', Simisola Aremo' and Olaperi Sowunmi ${ }^{2}$ \\ 'Department of Computer and Information Science, Covenant University, Ota, Nigeria; \\ sena.okuboyejo@covenantuniversity.edu.ng, aremo.simisola@gmail.com \\ ${ }^{2}$ School of Computer Science and Applied Mathematics, University of the Witwatersrand, South Africa; \\ olaperi.okuboyejo@wits.ac.za
}

\begin{abstract}
Objective: Time management methods need to be employed to encourage students in time tracking. This work presents the development and implementation of a task management application for task and time management. Methods: The functionalities of the task manager were modelled using UML (Unified Modelling Language), and a prototype of the system developed using Android Studio as the code compiler for the mobile application. A pilot test was carried out to evaluate ease of use, usefulness and continuous usage of the application. Findings: Although some of the respondents had found the application useful in helping them track their time and monitor their tasks, they did not visit the application frequently hence, no desirable affect on the outcome of their time management skills. Application: The strategic application of this solution to a wider audience would also increase productivity within related demographics such as among business owners, busy executives and parents.
\end{abstract}

Keywords: Academic Performance, Education, Students, Task Manager, Time Management

\section{Background Information}

Time is referred to as one of the most important resources to man and it is a very important ingredient to productivity; therefore, a person's proficiency to manage time effectively and efficiently cannot be exaggerated. Time management can be summarized as skillfully making the best use of time to accomplish a goal or task. It directly affects overall productivity as it determines the amount of effort and creativity invested into the work to be done.

Task management simply involves managing a task throughout its lifespan. The lifespan of a task begins from an inactive (ready) state and continues through several other states (delegated, rejected, assigned, terminated, expired, forwarded, finished, failed) but essentially concludes when the task has been completed. It involves planning, testing, tracking, reporting and effectively managing all aspects of a task, including its status, priority, time, human and financial resources assignments, recurrence, and notifications. Task management is very important in the working environment as it can help either individuals or a group of people achieve goals. Furthermore, ability to keep track of tasks deadlines, meetings and project team management makes the task management crucial for a busy knowledge worker.

In an academic setting, time management is just as important. Undergraduate university students, whether full time or part time, are also faced with the responsibility of organizing and managing their time commitments and effective time management is directly responsible for the positive mental and academic performance of the students. As the academic session progresses, these commitments become cumbersome and could weigh down on the students' productivity if not managed properly. In recent times, there has been a high incidence of poor academic performance by university undergraduates. This has been due, in the most part, to poor time management. When time is not effectively managed, important commitments (which include attending lectures, submitting assignments/projects and participating in tests and examinations) necessary for academic success are compromised. This eventually leads to imbalance, encourages 
malpractice, and in extreme cases, could cause several emotional, psychological and health problems.

In a bid to assist, workshops, one-on-one counselling sessions as well as the use of media materials (magazines, pamphlets, books and tapes) have been utilized. However, they have some disadvantages. Workshops might be labor effective and cover wide ratio of students, but there is little assurance to the impact that this measure will have on students' performance. On the other hand, one-on-one counselling sessions make up for the effectiveness that the workshops might not be able to provide but they are labor intensive and might not cover a wide ratio of students. Therefore, new measures need to be introduced into the academic system to encourage development of time management skills and ensure improvement of student's performance. This is where the task manager comes in to the scene, serving as a helper to assist the student in managing some of the tasks that would be referred to as less pressing; leaving enough room for the student to handle more pressing tasks that would require full attention 1 .

Since the birth of Artificial Intelligence (AI) in 1956, remarkable achievements which have transformed people's lives have been reached. One of such achievements is the invention of software agents. Software agents are programs that act on behalf of or with a user or another program. These software agents can make decisions independently but are usually constrained by other agents and software in the environment. A task manager is a scheduling agent which is a type of software agent. The task manager does the job of aiding its user in managing time commitments and performing tasks. The framework of the task manager is made up of a to-do list, a calendar, a reminder and a task executor. The aim of this work therefore is to design, implement, and pilot-test and evaluates a prototype task manager to assist university students with effective time management. The evaluation will attempt to answer the following research questions (RQ):

RQ 1: Was the system easy to use (learn ability and Navigation)?

RQ 2: Was the system useful in helping manage your academic tasks?

After an extensive literature review, the functionalities of the task manager were modelled using UML (Unified Modelling Language), and a prototype of the system developed using Android Studio as the code compiler for the mobile application. By carrying out this study, undergraduate students would be able to manage their time effectively as well as monitor their daily habits and revise them if necessary. In addition, the strategic application of this solution to a wider audience would also increase productivity within related demographics such as among business owners, busy executives and parents.

\subsection{Existing Works}

A task manager, otherwise known as a To-do list manager is a personal assistant that performs the job of planning, sorting, notifying, delegating and in some cases executing a set of user's tasks ${ }^{2}$. Its purpose is to aid the user in handling time commitments and reducing stress caused by cognitive overload. Task managers provide knowledge workers with a workspace to manage projects, tasks and to-dos individually or as part of a team. $\operatorname{In}^{3}$ a Task Gallery, a Window manager was developed. It uses 3D graphics and redirection mechanism to support document comparison and task management. Scenario Analysis was also recommended by ${ }^{4.5}$ as a useful tool for task managers working in environments where long-term thinking, flexibility and collaboration are critical.

In the academic environment, the task manager reminds the student of upcoming scheduled calendar events such as lectures and overdue tasks, notifies the student when a task to be completed becomes overdue thereby making it inconvenient to procrastinate and organizes tasks in the to-do list in order of importance. For the sake of clarity, events are occasions which have with fixed information (start time, end time, date, and location), an examination taking place from 2:00 pm to 4:00 pm, a lecture holding from 8:00am to 10:00am, are examples of events in an academic environment. Myers briefly describe the terms events and tasks; events are usually created on the task manager's calendar with each of these engagements having fixed start times and end times which is important to their classification as events. Tasks on the other hand, are not classified by their start times and end times, they are however classified by their priorities $\frac{6,7}{}$. Tasks are created on the task manager's to-do list which indicates basically, the name of the task and the priority of the task. Some task managers also perform autonomous actions for the student such as sourcing for useful information from the internet as related to an assignment, generating a map with the shortest possible distance to the event location and suggesting suitable study times based on the workload of the academic calendar. Although, these are just a few assistive behaviors, the possibilities of the task manager are endless. 
While some task managers exist, they have some limitations. My Study Life is a planner application developed for students, teachers and lecturers to assist with time management through the study plan of the student (https:// www.mystudylife.com). The application allows the user to store your classes, homework and exams in the cloud making it possible for the user to sync his data between any of his devices. It allows the user to view homework due and overdue for classes, classes which conflict with your exams and add revision tasks for a specific exam. It can be viewed in a week-view calendar however since it uses cloud storage; it requires login information which includes the email address and password to be authenticated first before the user can gain access.

Planner Plus is a productivity application for organizing calendar schedules and ultimately for time management (http://www.appxy.com/planner-plus/). It uses the concept of events and tasks; each event has a title, a time window (that is, a start time and an end time), a location with the option of whether to invite people or not while each task on the other hand has the title of the task, the date the task is to be performed and its priority level. It allows the user to sync the calendar schedule created with other calendars like the Google Calendar and allows the user to view the application in a month view.

TimeTune Schedule Planner is a productivity application built for time management (http://timetune.center/). The application can be conveniently used as a daily task reminder, daily activity manager, student calendar, timetable manager, schedule organizer, daily routine optimizer, time manager or daily planner. However, it does not make use of a calendar as a schedule technique but instead uses a concept of repetitive activities also known as routines. Routines defined in the application can be daily routines or weekly routines, but the application only has a limit of 15 days. The routine schedules work like a calendar but you don't need to enter calendar dates because a calendar is not needed since all the days of the week have been taken care of. The application also has a routine statistic where it analyses the users' distribution of time based on the routine schedule in a quantitative format. It also calculates the duration spent on an activity based on the start time and end time defined by the user. The goal of the application is to help the user with time management using repetitive routine tasks instead of a calendar, so it does not clutter your calendar schedule.

\section{Methodology}

\subsection{System Requirements}

The academic task planner is a mobile application which allows the students to access it on the go as required. The functional requirements of the system include:

The user shall be able to create, update or delete events in the calendar view.

The user shall be able to create, update and delete tasks (to-dos) in the calendar view.

The user shall be able to view created events and tasks (to-dos).

The user shall be able to choose when to delegate time allocation (scheduling) of to-dos to the system.

The system should be able to search for available time for to-dos based on user-defined constraints.

The system should respond with a message box when no available time for a to-do has been found.

The system should be able to notify the user when an event almost due.

Non-functional requirements on the other hand are indirectly concerned with the services offered by the system. They instead define the constraints of the system and not individual functionalities. For the system, the following non-functional requirements were defined:

The size of the system shall be moderate to allow it run smoothly on the mobile device without causing an overload on the processor.

The system shall have a clean and friendly user interface with easily understandable navigations.

The system shall be compatible with all students' mobile devices allowing for easy portability.

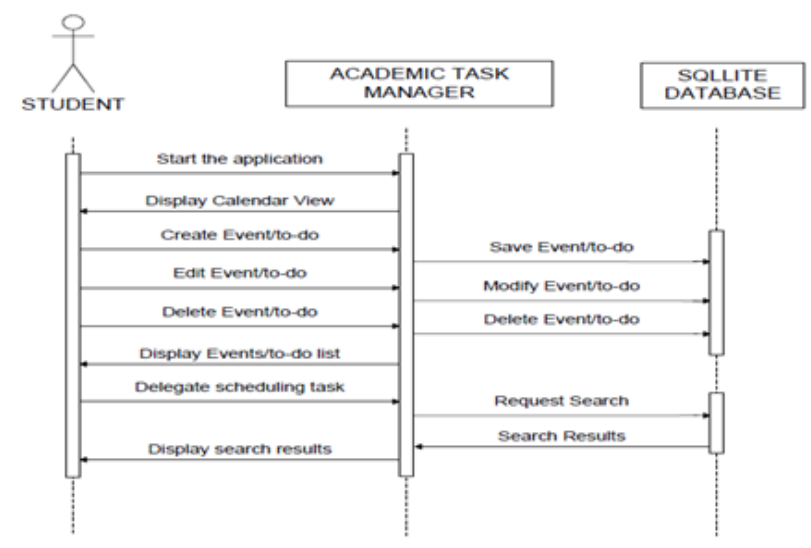

Figure 1. Sequence Diagram. 


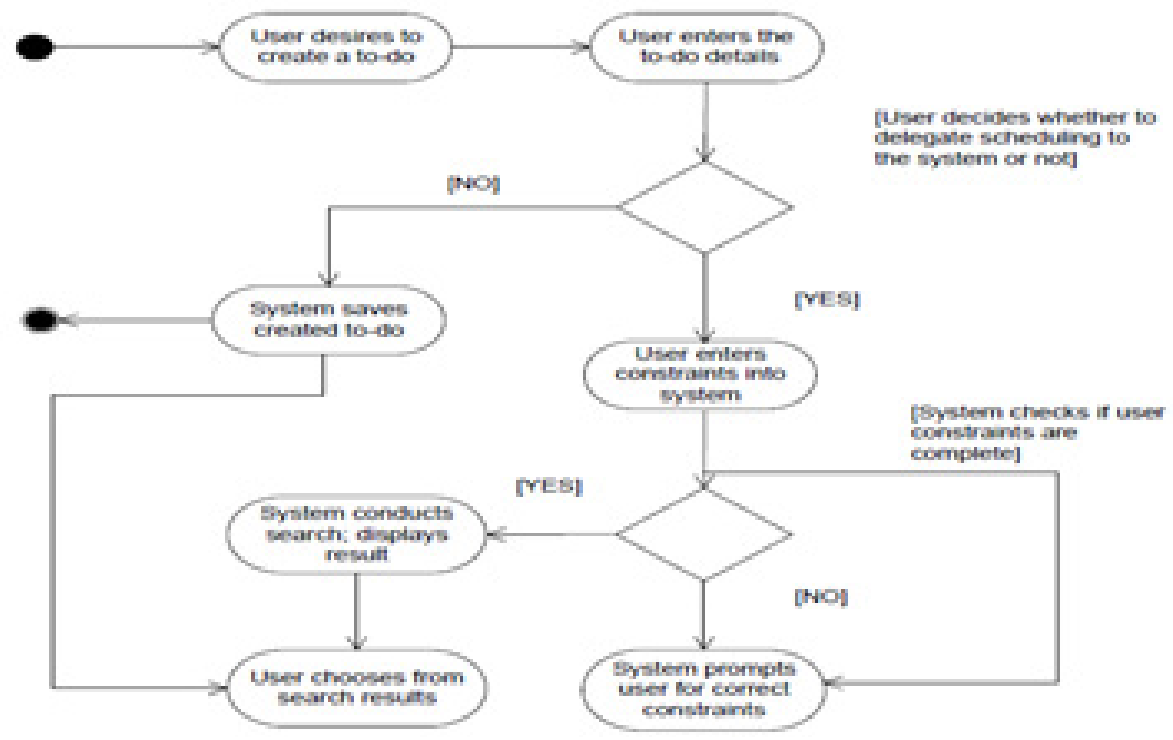

Figure 2. Activity Diagram.

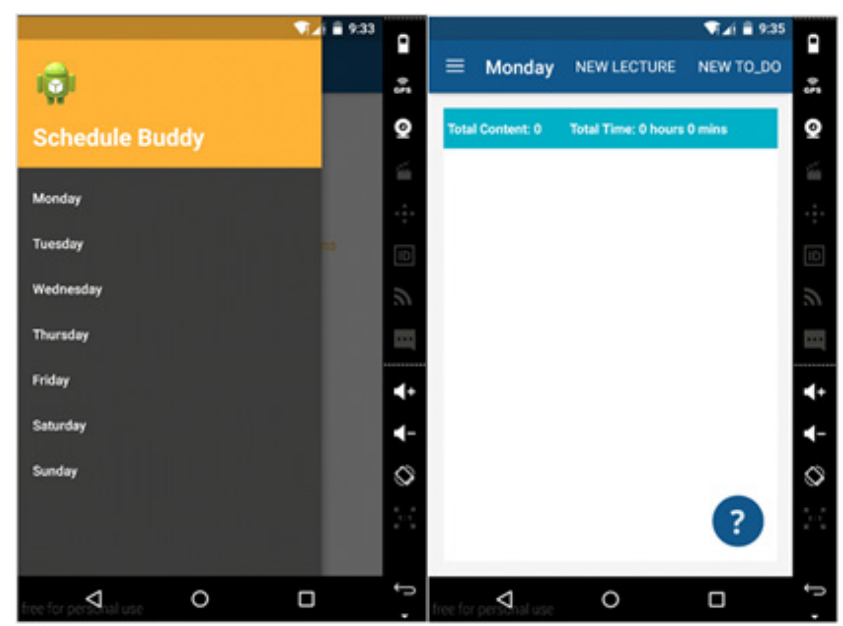

Figure 3. Screen shot for the menu and day of the week.

The system design was modelled using UML. The sequence and activity diagrams are represented in Figures 1 and 2 respectively. The sequence diagram shows the interaction of the various components of the system while the activity diagram shows the flow of interaction while using the system.

Several tools where employed in the development of the system, they include the Java programming language, Android studio and MySQLite database. Android Studio is the official IDE for Android app development; it offers features that enhance productivity when building Android apps. SQLite is embedded into the Android platform as it was used as database engine.

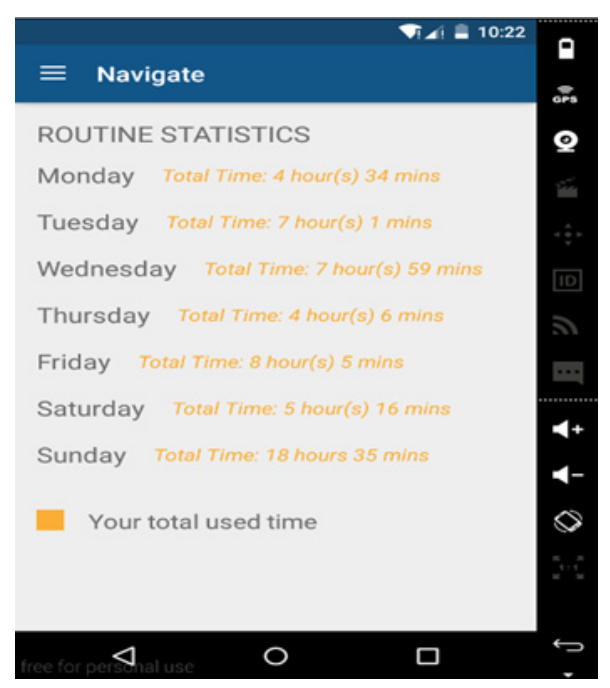

Figure 4. Screenshot for routine statistics for a week.

\subsection{System Implementation}

The application opens by showing the statistics of the user's routine tasks for each week day. Contained in the action bar are: the title of the day, a link to create a new lecture and a link to create a new "to-do". It also contains a menu navigation bar which shows the list of week days from Monday to Sunday. Each day view shows the number of items that the user wants to do and the overall amount of time that it would take to complete each task. This is shown in Figure 3 and Figure 4 shows the screenshots of routine statistics for the week. 


\subsection{System Pilot Test and Evaluation}

After the academic task manager application had been developed, a pilot test was carried out to evaluate its ease of use, usefulness and continuous usage. It was installed and deployed into the student's tablet devices to allow them to interact with it. The students' experiences were captured using online surveys. Twenty-five (25) final year students participated in the survey from a convenience sample, but only 17 responses were received, which made up $68 \%$ of the total population of respondents.

\section{Results}

RQ 1: System Learnability and Ease of Use

Respondents were asked whether the application had been learnable and easily navigable. $76.48 \%$ of the total respondent population agreed that the application was indeed learnable, $11.76 \%$ had disagreed that the application had been learnable while the remaining $11.76 \%$ had been indifferent to the question.

\section{RQ 2: System Usefulness}

The respondents were asked if the application had been useful in helping them arrange their tasks and track their time usage; $47.06 \%$ of the respondents had found it useful for arranging their tasks and keeping track of their time, $29.41 \%$ disagreed to its being useful while the remaining $23.53 \%$ of the total population of respondents were indifferent to the question.

On the frequency of use, $11.76 \%$ of the total respondents used the deployed application often, $64.71 \%$ of the total respondents did not refer to the application again after its first use while the remaining $23.53 \%$ of the total respondents were altogether indifferent about the application. In summary, although some of the respondents had found the application useful in helping them track their time and monitor their tasks, they did not visit the application frequently and so it could not make a desirable effect on the outcome of their time management skills.

\section{Conclusion}

Effective time and task management has proven to be a skill that affects undergraduate student's performance as well as businesspeople and busy executives. It is a quality that should be best developed from the undergraduate level therefore making it important to create an awareness of its necessity. Its absence causes an academic imbalance, encourages malpractice and ultimately leads to poor performance. Time management methods need to be employed to encourage students to track their use of time in carrying out their daily tasks. The Academic Task Manager assists the undergraduate student to manage their time effectively particularly for those with poor time management skills and to encourage them to monitor their daily habits and revise them if necessary. The results from the pilot evaluation will shape future modifications to the system especially in designing features to enhance frequent usage of the system, which will be tested on a large scale.

\section{References}

1. Budiman R. Utilizing Skype for Providing Learning Support for Indonesian Distance Learning Students: a Lesson Learnt. Procedia-Social Behavioral Science. 2013; 83(5):5-10.

2. Premawardhena NC. Introducing computer aided language learning to Sri Lankan schools: Challenges and perspectives. 2012 15th International Conference on Interactive Collaborative Learning (ICL). 2012; p. 1-5.

3. Robbins DC, Robertson G, Van Dantzich M, Robbins D, Czerwinski M, Hinckley K. The Task Gallery: A3D Window Manager. Research Gate. 2000; 2(1):1-9.

4. Krueger RA, Casey MA, Donner J, Kirsch S, Maack JN. Social Analysis Selected Tools and Techniques. Social Development Papers. The World Bank. 2001; p. 1-93. PMid:11532199 PMCid:PMC48148

5. Postma TJBM, Liebl F. How to improve scenario analysis as a strategic management tool? Technological Forecasting and Social Change. 2005; 72(2):161-73. https://doi. org/10.1016/S0040-1625(03)00152-5

6. Stevens R, Goble C, Baker P, Brass A. A classification of tasks in bioinformatics. Bioinformatics. 2001; 17(2): 180-8. https://doi.org/10.1093/bioinformatics/17.2.180 PMid:11238075

7. Weir CR, Nebeker JJR, Hicken BL, Campo R, Drews F, LeBar B. A Cognitive Task Analysis of Information Management Strategies in a Computerized Provider Order Entry Environment. Journal of the American Medical Informatics Association. 2007; 14(1):65-75. https://doi.org/10.1197/ jamia.M2231 PMid:17068345 PMCid:PMC2215066 